\title{
Do isoluminant color changes capture attention?
}

\author{
ANTHONY LAMBERT, IAN WELLS, and MATTHEW KEAN \\ University of Auckland, Auckland, New Zealand
}

\begin{abstract}
Four experiments are reported in which the effects of peripheral cues on visual orienting were investigated. In the luminance condition, the cues consisted of a peripheral change in stimulus luminance. In the isoluminance condition, the cues consisted of an isoluminant color change, using the transient tritanopic technique. In Experiments 1 and 2, it was found that peripheral luminance cues captured attention, whereas peripheral isoluminance cues did not. In Experiments 3 and 4, the participants detected a peripheral target that was also isoluminant with the background. Under these conditions, it was found that both luminance and isoluminance cues captured attention. The results are discussed in terms of the roles of the dorsal and ventral streams in visual orienting, and it is concluded that our findings provide partial support for the contingent involuntary orienting hypothesis of C. Folk and colleagues.
\end{abstract}

Do isoluminant visual stimuli capture attention? The question appears simple and amenable to a straightforward scientific answer. In principle, all one should need here is an experimental paradigm for assessing attentional capture (see, e.g., Jonides, 1981; Posner \& Cohen, 1984), together with a technique for equating the luminances of stimulus objects. Unfortunately, previous attempts to address this issue have not produced a clear-cut answer, and the literature on this question can only be described as equivocal. In this paper, we will describe four experiments in which a more definitive answer was sought. In comparison with earlier investigations, we employed different techniques, both for assessing attentional capture and for rendering stimuli isoluminant. Before describing these experiments, two further issues need to be reviewed: Why are isoluminant stimuli interesting, and what is the status of current evidence concerning attentional capture by isoluminant visual changes?

\section{Why Are Isoluminant Stimuli Interesting?}

Interest in the processing of isoluminant stimuli stems chiefly from the relation between isoluminance and the idea that visual perception arises from the combined operation of two distinct processing pathways. According to Ungerleider and Mishkin (1982), the ventral stream originates in parvocellular layers of the lateral geniculate nucleus (LGN) of the thalamus and proceeds to the inferotemporal cortex via the primary visual cortex and the ventral prestriate cortex. A primary function of the ventral

\footnotetext{
We acknowledge the assistance of Barry Hughes in running Experiment 1 . We thank Mike Corballis for valuable discussions concerning the tritanopic technique. We also acknowledge the support of the Auckland University Research Committee. Correspondence concerning this article should be addressed to A. Lambert, Research Centre for Cognitive Neuroscience, Department of Psychology, University of Auckland, Private bag 92019, Auckland, New Zealand (e-mail: a.lambert@auckland. ac.nz).
}

stream is thought to be the encoding of the identity of visual objects. Hence, it is sometimes referred to as the what system. In contrast, the dorsal stream originates in magnocellular layers of the LGN and proceeds to the posterior parietal cortex via the primary visual cortex and the dorsal prestriate cortex. The encoding of spatial information, including information required for appropriate orienting, is thought to be a primary function of the dorsal stream. Hence, it is sometimes known as the where system. An interesting feature of cells in the dorsal or magnocellular processing pathway is that they are highly responsive to luminance changes but unresponsive to changes in the color, or chrominance, of objects within their visual field. Cells in the ventral/parvocellular pathway respond to both luminance and chrominance changes (Livingstone \& Hubel, 1988). Research on this topic, largely prompted by the findings and proposals of Ungerleider and Mishkin and of Livingstone and Hubel, has suggested a promising convergence between perceptual, psychophysical, physiological, and neuropsychological observations. For example, physiological and neuropsychological evidence has suggested an association between the dorsal-magno pathway and motion perception (Livingstone \& Hubel, 1988; Zihl, von Cramon, \& Mai, 1983). Consistent with this, perceptual and psychophysical work has shown that the perceived motion of a moving grating is markedly impaired for isoluminant stimuli when the grating is defined purely by color variation (Livingstone \& Hubel, 1988). According to this logic, observing the behavioral correlates of luminant and isoluminant stimuli can be valuable in diagnosing whether a particular perceptual property is encoded within the dorsal stream. In the context of an interest in the property of attentional capture, this enables one to pose an important theoretical question. If the capture of attention by changes in visual stimulation is mediated solely by processing within the dorsal stream, isoluminant visual changes will fail to capture attention. Alternatively, if both luminant and isoluminant visual 
changes can be observed to capture attention, this can be interpreted as evidence that attentional capture can be mediated by the ventral stream, as well as by the dorsal stream. Following the logic just described, one might conceivably also interpret this pattern as evidence that attentional capture might be mediated entirely within the ventral stream. However, a substantial body of neuropsychological evidence suggesting an association between spatial attention and the posterior parietal cortex renders the latter interpretation implausible (Robertson \& Rafal, 2000).

\section{Previous Evidence on Attentional Capture by Isoluminant Stimuli}

As was mentioned earlier, previous work on this issue presents a rather confused picture. Theeuwes (1995) reported a series of visual search experiments in which observers viewed arrays of green outline circles containing red line segments. In the search display, the observers were presented with a new element that was identicalin color to items in the initial display. The task was to indicate the orientation of the inner red line segment in the new item. Theeuwes found that search time was independent of display size when the target item differed from the distractors in terms of luminance. This was taken to indicate parallel and preattentive processing of the target stimulus, a pattern commonly referred to as pop-out search. In contrast, when the target was rendered isoluminant with the distractors, serial functions were obtained, indicating serial attentive search. Theeuwes's carefully designed experiments suggested that even when observers are set to search for the new object in a display, the new object does not pop out under isoluminant conditions. However, at about the same time, Yantis and Hillstrom (1994) published a series of experiments suggesting a diametrically opposite conclusion: that the appearance of a new object that is isoluminant with the background does capture attention. Yantis and Hillstrom viewed their experiments as involving a test between two alternative hypotheses concerning the mechanism of attentional capture. According to the luminance increment hypothesis, luminance change is a necessary condition for attentional capture. This hypothesis would, of course, also be consistent with mediation by the dorsal stream. According to the new-object hypothesis, attentional capture is triggered by the appearance of a new perceptual object. This hypothesis would be consistent with mediation by the ventral stream. In the experiments, target and distractor objects were distinguished from the background in terms of equiluminant discontinuities in texture, motion, or stereoscopic depth. Under these conditions, relatively flat search functions were observed for new target objects, suggesting that attentional capture is driven by onset of a new perceptual object and that luminance change is not a necessary precondition for attentional capture. However, the design of Yantis and Hillstrom's experiments was cogently criticized by Gellatly, Cole, and Blurton (1999), who pointed out that although Yantis and Hillstrom's stimuli were equiluminant with their backgrounds within a single frame of the display sequence, their design failed to equate the temporal dynamics of luminance change across successive display frames. In their Experiments 3 and 4, Gellatly et al. controlled for dynamic changes in luminance and examined visual search functions for motion-defined targets. Under these conditions, they found that the attentional advantage enjoyed by novel targets was substantially attenuated, in comparison with the results of Yantis and Hillstrom, but was not entirely abolished. From this, they concluded, in opposition to Yantis and Hillstrom, that "attention is not reliably captured at the level of object representations" (p. 1619). The residual advantage for novel targets was explained by proposing that attentional capture can be driven by detection of sensory change across several different stimulus dimensions. Although it is clear that luminance change is a particularly potent factor capable of capturing attention, Gellatly et al. proposed that attention may also be captured by changes in other sensory dimensions, including second-order stimulus properties, such as direction of motion. In parenthesis, it is worth noting that this mechanism would also be consistent with dorsal stream mediation of attentional capture. In their Experiment 2, Gellatly et al. examined search functions for isoluminant chromatically defined targets and found a substantial attentional advantage for novel target objects. They concluded that isoluminant color changes are capable of capturing attention - a proposal that is, of course, inconsistent with the hypothesis that attentional capture is mediated exclusively within the dorsal stream. However, the task of rendering stimuli chromatically distinct yet isoluminant is far from straightforward. In this case, the procedure involved ensuring that the measured ( physical) on-screen luminances of the stimuli $\left(36.0 \mathrm{~cd} / \mathrm{m}^{2}\right)$ and the background $\left(36.4 \mathrm{~cd} / \mathrm{m}^{2}\right)$ were approximately equivalent. As Gellatly et al. acknowledged, this procedure failed to guarantee that their stimuli were isoluminant with respect to subjective luminance or neural responses within luminance-sensitive neural pathways. This methodological problem weakens their conclusion that isoluminant color change can capture attention, especially when considered alongside findings that even slight changes in luminance can capture attention (Lambert, Naikar, McLachlan, \& Aitken, 1999; Theeuwes, 1995) and in light of the possibility that luminance-sensitive pathways subserving spatial orienting in the dorsal stream may enjoy intimate interaction with color-sensitive pathways contributing to object identification within the ventral pathway (see Merigan \& Maunsell, 1993).

In view of the above, the question of whether isoluminant visual changes, in general, and isoluminant color changes, in particular, capture attention remains undecided. In the experiments reviewed above, attentional capture (Gellatly et al., 1999; Yantis \& Hillstrom, 1994) and the related concept of attentional pop-out (see Theeuwes, 1995) were indexed by examining the slope of functions relating response time to display size in visual search tasks. However, as many readers of this journal will be aware, 
the concept of attentional capture is associated not only with the visual search paradigms described above, but also with the attentional effects of peripheral, or exogenous, cues (see, e.g., Jonides, 1981; Luck \& Thomas, 1999; Posner \& Cohen, 1984). In this paradigm, participants respond to target objects under conditions of spatial uncertainty. Prior to the target, a pretrial cue is presented at one of the potential target locations. Performance on trials in which the target appears at the cued location can be compared with that on uncued trials, in which the target appears elsewhere. Facilitation of the former, as compared with the latter, may be viewed as an index of the extent to which attention has been drawn to a location in response to onset of the cue stimulus. A number of findings are consistent with the view that, in this paradigm, peripheral cues capture attention in a rapid, reflexive, and automatic way. Following a peripheral cue, cued locations enjoy a processing advantage over uncued locations even when the delay between cue and target onset is very brief (Cheal \& Lyon, 1991; Muller \& Rabbitt, 1989), even when the cue is uninformative with respect to target location (Posner \& Cohen, 1984), and even when participants are instructed to ignore the cue (Jonides, 1981).

Given the confused nature of the findings generated from visual search studies, and since, to our knowledge, no previous experiments have examined attentional effects of isoluminant peripheral cues, we decided to employ the peripheral cuing paradigm in order to seek a clearer answer to the question of whether isoluminant color changes capture attention. In addition, we used a somewhat different technique for rendering our stimuli isoluminant. Cavanagh, Adelson, and Heard (1992) have described a multitude of difficulties that may be encountered in the course of attempting to construct stimuli that activate chromatic pathways but remain invisible to the luminance pathway. Indeed, according to Cavanagh et al., the creation of truly isoluminant stimuli in complementary colors appears to be virtually impossible using standard computercontrolled cathode ray tube (CRT) displays (see pp. 219220). Fortunately, Cavanagh et al. described a way to circumvent these problems by using tritanopic stimuli. These are created by superimposing a high-intensity yellow adapting field onto a deep blue stimulus. Under these conditions, the luminance pathway is strongly driven by the yellow adapting field (i.e., by the combined activity of the $\mathrm{R}$ [red-sensitive] and the $\mathrm{G}$ [green-sensitive] cones). However, the B (blue sensitive) cones are thought to have only a very weak input to the luminance pathway. Hence, in a tritanopic display, the B cones encoding the blue image will activate the chromatic pathway but will exert little or no effect on the already active luminance pathway. That is, the spatial variation in the blue image will be encoded by the chromatic pathway but will be invisible to the luminance pathway. This is termed a tritanopic stimulus, since it would be invisible to a tritanope (a person who lacks B cones). Hence, according to Cavanagh et al., the tritanopic technique provides a way of constructing stimuli that are isoluminant or near isoluminant and that, therefore, offer a means of isolating the contribution of color-sensitive and luminance-sensitive perceptual pathways.

In the experiments described below, we compared attentional effects of peripheral cues rendered isoluminant via the tritanopic technique with attentional effects of luminance-based peripheral cues. Since the tritanopic technique rendered our cue stimuli relatively subtle and difficult to perceive, we were concerned that this comparison might be confounded by stimulus salience. That is, reduced or absent attentional capture effects might be attributed to the reduced salience of the isoluminant cue stimulus, rather than to its isoluminance per se. In order to control for this, the participants performed two tasks: an attentional cuing task, in which target detection latency at cued and uncued locations was assessed, and a cue discrimination task, in which the ability to discriminate whether a cue stimulus had been presented on the left or the right of the display was assessed. The aim of the latter task was to test for differences in the salience (i.e., discriminability) of the isoluminant and the luminance-based cue stimuli.

\section{EXPERIMENT 1}

In Experiment 1, we examined the attentionaleffects of uninformative peripheral cues that were defined either by a luminance change or by an isoluminant color change.

\section{Method}

Participants. Sixteen adult volunteers took part. There were 8 participants in the isoluminance condition and 8 participants in the luminance condition.

Apparatus. The experiment was performed using 16 IBMcompatible 486 PCs, together with 15 -in. color monitors. The visual display and timing software for the experiment was written in Turbo Pascal version 7.0. VGA resolution $(640 \times 350$ pixels $)$ was used. The testing was performed in a teaching laboratory, with normal levels of room illumination.

Display and Stimuli (luminance condition). The fixation display comprised a central cross $(0.4 \times 0.4 \mathrm{~cm})$ and two outline squares $\left(4.2 \mathrm{~cm}^{2}\right)$ to the left and right of the cross. The lines forming each square were $2 \mathrm{~mm}$ (4 pixels) thick. The inner edge of each square was $4.0 \mathrm{~cm}$ from fixation. The target stimulus was a filled square, $0.4 \times 0.4 \mathrm{~cm}$, presented at the center of either the left or the right outline square. The inner edge of the target was $5.9 \mathrm{~cm}$ from fixation. The software controlling the experiment allowed black/ gray/white levels to be varied in 64 steps from 0 (pure black) to 63 (pure white). The fixation cross, outline squares, and target stimuli were all presented in mid-gray (gray value $=30$; luminance $=$ $30 \mathrm{~cd} \mathrm{~m}^{-2}$; CIE L*a*b coordinates $\left.=73.5,-6.4,-4.4\right)$ against a black background. Cue stimuli were created by the transient brightening (for $66 \mathrm{msec}$ ) of the central portions of the outline square on the left or the right of the display. This was achieved by presenting a light gray outline square, formed by lines $1 \mathrm{~mm}$ ( 2 pixels) thick, against the background of either the left or the right gray square. The gray level used to form the light gray cue stimuli was varied across trials and could take three values: 32,35 , and 40 . These will be referred to below as Luminance Cue Level One, Luminance Cue Level Two, and Luminance Cue Level Three, respectively. The luminances and CIE L*a*b coordinates were as follows: Cue Level One, lumi- 
nance $=34 \mathrm{~cd} \mathrm{~m}^{-2}, \mathrm{CIE} \mathrm{L} * \mathrm{a} * \mathrm{~b}=75.2,-6.5,-4.5$; Cue Level Two, luminance $=39 \mathrm{~cd} \mathrm{~m}^{-2}$, CIE L*a*b $=78.1,-6.7,-4.7$; Cue Level Three; luminance $=49 \mathrm{~cd} \mathrm{~m}^{-2}$, CIE L*a*b $=79.0,-6.8,-4.7$. Viewing distance was approximately $55 \mathrm{~cm}$.

Display and Stimuli (isoluminance condition). The spatial dimensions of the stimuli in this condition were the same as those for the luminance condition. In this case, the stimuli were presented against a yellow background. The experimental software allowed the intensity of the red, green, and blue (RGB) components of any color to be varied from 0 to 63 units. The yellow display background was formed by combining 50 units of red with 50 units of green (luminance $=80 \mathrm{~cd} \mathrm{~m}^{-2}$, CIE L*a*b $\left.=87.3,-26.5,81.1\right)$. The fixation cross, the target, and the $2-\mathrm{mm}$ outline squares that were permanently present on the display were presented as a slightly brighter yellow against the yellow background. This brighter yellow (luminance $=96 \mathrm{~cd} \mathrm{~m}^{-2}$, CIE L*a*b $=91.4,27.5,84.4$ ) was formed by combining 56 units of red with 56 units of green. The cue stimuli were formed by presenting a thin blue outline square (1-mm thickness, 2 pixels) against one of the bright yellow outline squares that were permanently on the display. This thin outline square was formed by adding 32,35 , or 40 units of blue. The addition of blue against a bright yellow background did, of course, make the cue stimuli appear as white against yellow. The stimuli so formed will be referred to below as Isoluminance Cue Level One, Isoluminance Cue Level Two, and Isoluminance Cue Level Three, respectively. The CIE L*a*b values and the physically measured luminances of these cue stimuli were as follows: Isoluminance Cue Level One, luminance $=95 \mathrm{~cd} \mathrm{~m}^{-2}, \mathrm{CIE} \mathrm{L} \mathrm{a}^{*} \mathrm{~b}=93.0,-15.9,20.6$ ); Isoluminance Cue Level Two, luminance $=94 \mathrm{~cd} \mathrm{~m}^{-2}$, CIE L*a*b $=$ 93.1, $-14.8,16.7$; Isoluminance Cue Level Three, luminance $=$ $93 \mathrm{~cd} \mathrm{~m}^{-2}$, CIE L*a*b $=93.3,-13.1,10.7 .^{1}$

Validation of isoluminance condition. In the isoluminance condition, the phenomenal appearance of the cues was as follows. They underwent a transient but perceptible color change from yellow to white, but unlike the luminance cues, they did not appear to "flash." In order to check that the cues in this condition achieved isoluminance with the background, a flicker procedure was performed. On each trial of this procedure, the observers fixated the central cross while cue stimuli were presented repeatedly within both the left and the right outline boxes. The cue stimuli were switched on for $50 \mathrm{msec}$ and off for $50 \mathrm{msec}$, over a 1-sec period (i.e., $10 \mathrm{on} / \mathrm{off}$ cycles were presented). The observers adjusted the intensity of the bright yellow outline squares, which formed the background against which the cues were presented. At both high and low intensities of yellow, the repeatedly presented cues appeared to flicker. The arrow keys on the keyboard were used to adjust the intensity of the yellow outline squares until the flicker associated with repeated cue presentation was eliminated. Two observers performed this procedure 12 times each -4 times for each of the three levels of isoluminance cue. On half of these trials, the observer began by viewing yellow outline squares that were clearly higher in luminance (red, 60 units; green, 60 units; blue, 0 units) than the cue (red, 56 units; green, 56 units; blue, 32, 35, or 40 units). On the remaining trials, the observers began by viewing a yellow outline square that was clearly lower in luminance (red, 52 units; green, 52 units; blue, 0 units) than the cue stimulus. Pressing the up-arrow or the down-arrow key caused an increase (or decrease) of one unit in the intensity of the red and green light that formed the yellow outline square. After each adjustment was made, there was a delay of $2 \mathrm{sec}$ before the next trial began. The mean intensity of red/green that was judged as isoluminant with Cue Level One (red, 56 units; green, 56 units; blue, 32 units) was $56(S D=0)$. The mean intensity of red/green that was judged as isoluminant with Cue Level Two (red, 56 units; green, 56 units; blue, 35 units) was $56(S D=0)$. The mean intensity of $\mathrm{red} / \mathrm{green}$ that was judged as isoluminant with Cue Level Three (red, 56 units; green, 56 units; blue, 40 units) was $56.25(S D=0.43)$. Thus, in all three cases, the intensity of yellow that was judged as isoluminant with the cue was identical or nearly identical with the yellow that was actually employed in the experiment (i.e., red, 56 units; green, 56 units; blue, 0 units). This suggests that the tritanopic technique was successful: The subjective brightness of the cue stimuli was identical or nearly identical with the yellow background against which they were presented.

Procedure (spatial cuing task). The same procedure was followed in the luminance and the isoluminance conditions. The participants were instructed to press the space bar as soon as they saw the target stimulus appear on the left or the right of the display screen. The participants were instructed to fixate the central cross throughout the experiment, and the importance of maintaining central fixation was emphasized. One second before the cues were presented, the central cross disappeared for $100 \mathrm{msec}$; that is, the central cross blinked at the beginning of every trial. This was done to draw the participants' attention to the fixation cross and to remind them to fixate centrally on every trial. A left or a right cue stimulus was then presented for $66 \mathrm{msec}$. On $89 \%$ of the trials, the cue was followed by a target stimulus.

On trials with a target stimulus, the delay between cue onset and target onset (stimulus onset asynchrony, SOA) was 100 or $600 \mathrm{msec}$. The target stimulus disappeared when the space bar was pressed. After an interval that varied randomly from 500 to $1,000 \mathrm{msec}$, the central cross blinked to signal the beginning of the next trial.

Eleven percent of the trials were catch trials, on which the cue stimulus was presented but there was no target. The participants were informed about the occurrence of these trials and were instructed to refrain from responding on them. They were informed that responding on these trials would result in presentation of the message "Warning! Catch Trial Error" for $1 \mathrm{sec}$ at the bottom of the screen. Similarly, the participants were requested to avoid making anticipatory responses and were informed that pressing the space bar before the target appeared would result in presentation of the message "Warning! Anticipation Error" for $1 \mathrm{sec}$ at the bottom of the screen.

At the beginning of the testing session, the participants were presented with 8 practice trials to familiarize them with the task. The participants then performed three blocks of 54 trials.

Procedure (cue discrimination task). The same procedure was followed in the luminance and the isoluminance conditions. The participants always performed the cue discrimination task after performing the spatial cuing task. The participants were instructed that, on each trial, the task was to decide whether a cue stimulus had been presented on the left or the right of the display and to indicate their response by pressing the left or the right arrow key on the keyboard. As in the spatial cuing task, the central cross blinked (for $100 \mathrm{msec}$ ) at the beginning of every trial. One second later, a cue stimulus was presented for $66 \mathrm{msec}$ on the left or the right of the display. Five hundred milliseconds after onset of the cue, the message "Side?" appeared $0.7 \mathrm{~cm}$ above the fixation cross and in the center of the screen. This was presented in the same shade of yellow/gray as the fixation cross and served as a signal for the participants to indicate, via the arrow keys, whether they believed the cue had been presented on the left or the right of the display. Immediately after the left or right arrow key had been pressed, a square box, identical with the target stimulus used in the spatial cuing task, was presented for $1 \mathrm{sec}$ on the same side as the cue stimulus. This served to provide the participants with feedback concerning the accuracy of their response. After offset of this feedback stimulus, there was an interval, which varied randomly between 500 and $1,000 \mathrm{msec}$, before the central cross blinked to signal the beginning of the next trial.

The participants performed 8 practice trials, followed by three blocks of 48 experimental trials.

Design (spatial cuing task). Each experimental session comprised 162 trials, which were divided into three blocks of 54 trials. There were 18 catch trials and 144 target trials. There were 72 cued trials, on which the target was presented on the same side of the dis- 
play as the cue, and 72 uncued trials, on which the cue and the target appeared on opposite sides of the display. There were 72 trials with a target on the left and 72 trials with a target on the right, and there were 72 trials with a brief $(100-\mathrm{msec})$ SOA and 72 trials with a longer (600-msec) SOA. Within each block, trial type varied pseudorandomly with respect to cue location, target location, SOA, and whether the trial was a target or a catch trial.

Design (cue discrimination task). Each testing session comprised 144 trials. There were 72 trials with a cue on the left and 72 trials with a cue on the right. There were 48 trials at each of the three cue levels. Trial type varied pseudorandomly with respect to side (left or right) and cue level.

\section{Results}

Spatial cuing task: Luminance condition. The mean rate of catch trial errors in this condition was $3.5 \%$. Response times less than $100 \mathrm{msec}$ in latency or greater than three standard deviations from the mean response time for that participant were excluded from further analysis. As a results of this procedure, $1.5 \%$ of the response times were discarded.

The response time results for the luminance condition of the spatial cuing task are shown in Figure 1. These data were entered into a three-factor analysis of variance for repeated measures. The factors were cuing (cued location vs. uncued location), SOA (100 vs. $600 \mathrm{msec}$ ), and cue level (One, Two, or Three). There was one significant effect in this analysis - the interaction between cuing and SOA $[F(1,7)=18.73, p<.005]$. At the short SOA, response times were significantly shorter when the targets appeared at the cued location $(313 \mathrm{msec})$, as compared with uncued location trials $[325 \mathrm{msec} ; F(1,7)=10.07$, $p<.025]$. At the long SOA, the difference between cued ( $313 \mathrm{msec})$ and uncued ( $306 \mathrm{msec}$ ) locations did not attain significance $[F(1,7)=2.80$, n.s. $]$. No other main effects or interactions approached significance in this analysis.

Spatial cuing task: Isoluminance condition. The mean rate of catch trial errors in this condition was $2.8 \%$. Response times less than $100 \mathrm{msec}$ in latency or greater than three standard deviations from the mean response

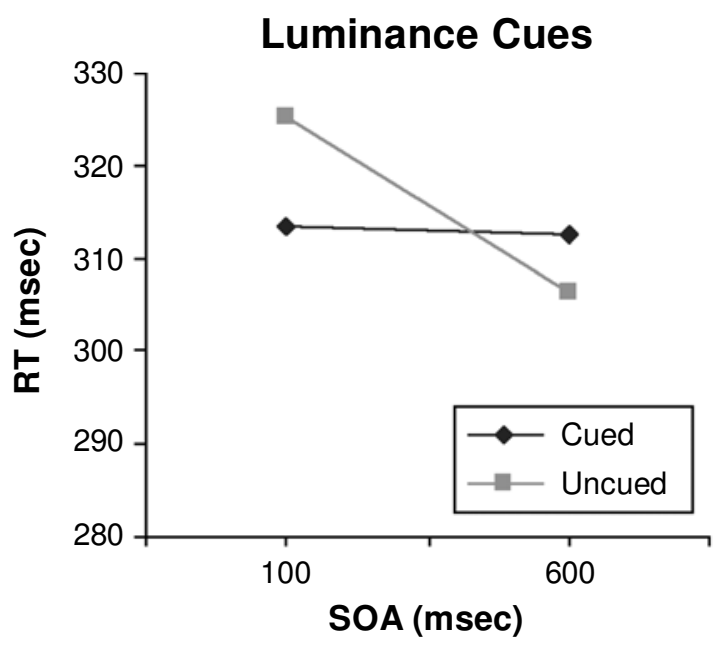

Figure 1. Response time (RT) results for the luminance condition in Experiment 1.

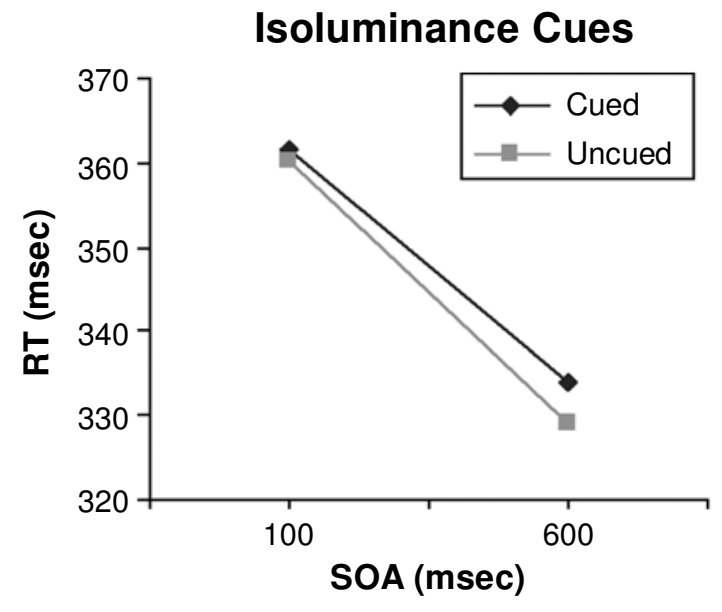

Figure 2. Response time (RT) results for the isoluminance condition in Experiment 1.

time for that participant were excluded from further analysis. As a result of this procedure, $2.6 \%$ of the response times were discarded.

The response time results for this condition are shown in Figure 2. These data were entered into analysis of variance, with the same factors as those for the luminance condition. Response times were shorter in the 600-msec SOA condition $(332 \mathrm{msec})$ than in the $100-\mathrm{msec}$ SOA condition [361 msec; $F(1,7)=23.73, p<.005]$. No other main effects or interactions were significant in this analysis. In particular, neither the main effect of cuing nor its interaction with SOA approached significance $(F<1)$.

Cue discrimination task: Luminance and isoluminance conditions. The participants' accuracy in the cue discrimination task is shown in Table 1. These data were entered into an analysis of variance with one repeated measures factor (cue level: One, Two, or Three) and one independent groups factor (cue condition: luminance cue vs. isoluminance cue). The main effect of cue level $[F(2,28)=$ $35.55, p<.001]$, the main effect of cue condition $[F(1,14)=$ $27.52, p<.001]$, and the interaction between these factors $[F(2,28)=28.18, p<.001]$ were all highly significant. As Table 1 shows, this interaction arose from the fact that performance was better in the isoluminance condition than in the luminance condition for Cue Levels One and Two. However, for Cue Level Three, performance in both conditions approached $100 \%$ and did not differ. ${ }^{2}$

\section{Discussion}

In the luminance condition, we observed the well-known and well-replicated attentional capture effect (Luck \& Thomas, 1999; Posner \& Cohen, 1984): At the short SOA, response latencies were significantly shorter when the targets appeared on the same side as the peripheral luminance change. In contrast, in the isoluminance condition, there was no evidence whatsoever of shorter latencies on cued trials $(362 \mathrm{msec})$ than on uncued trials $(360 \mathrm{msec})$. The failure of isoluminant peripheral color changes to 
Table 1

Cue Discrimination Accuracy (Percent Correct) in Experiment 1

\begin{tabular}{|c|c|c|c|c|}
\hline \multirow[b]{2}{*}{ Level } & \multicolumn{2}{|c|}{ Isoluminance Cues } & \multicolumn{2}{|c|}{ Luminance Cues } \\
\hline & $M$ & $S D$ & $M$ & $S D$ \\
\hline One & 96.1 & 4.7 & 60.2 & 15.3 \\
\hline Two & 97.9 & 2.9 & 88.3 & 10.9 \\
\hline Three & 98.2 & 1.7 & 97.4 & 3.5 \\
\hline
\end{tabular}

capture attention is not amenable to an explanation in terms of cue salience, with the isoluminance cues having lower salience than the luminance cues. Indeed, in the cue discrimination task, the participants' performance was significantly superior in the isoluminance condition. The accuracy with which the participants were able to discriminate the side of presentation for isoluminance cues approached $100 \%$ for all three cue levels. Despite this high level of discriminability, these cues failed to capture attention.

Whereas luminance cues produced attentional capture at the short SOA (shorter response latency on cued than on uncued trials), at the long SOA, there was no difference in response latency between cued and uncued trials in the luminance condition. A number of previous studies have also shown that the attention-capturing effect of a peripheral cue that is noninformative with respect to target location is short lived. Indeed, many studies have shown that under such conditions, at the long SOA, latencies are significantly longer at the cued than at the uncued location (Lambert, M. B. H. Spencer, \& Hockey, 1991; Maylor, 1985; Pratt, Hillis, \& Gold, 2001). This effect has been termed inhibition of return (IOR), reflecting an interpretation in terms of an inhibitory process acting against the return of attention to a recently inspected location. In the present study, although mean latencies were slightly longer on cued trials at the long SOA, the contrast between cued and uncued trials at this SOA did not attain significance. However, the absence of a significant IOR effect is tangential to the central concern of this study, which was with short SOA attentional capture effects in the luminance and the isoluminance conditions.

\section{EXPERIMENT 2}

In common with a number of earlier studies (e.g., Posner \& Cohen, 1984), the cues employed in Experiment 1 were uninformative with respect to target location. In agreement with earlier work, peripheral luminance cues captured attention even though they provided no information regarding target location (see above). However, effects of peripheral cues on visual orienting have also been studied in a slightly different situation in which the location of the peripheral cue provides useful information concerning the likely location of the target. In this situation, advantages for responding to targets at the cued location are typically observed across both short (e.g., 100-msec) and long (e.g., 600-msec) SOAs (Lambert, E. Spencer, \&
Mohindra, 1987). These effects are often interpreted in terms of the combined operation of both exogenous orienting (producing an advantage for the cued side at brief SOAs) and endogenous orienting (producing an advantage for the cued side at longer SOAs). Accordingly, the aim of Experiment 2 was to examine the effects on visual orienting of peripheral isoluminance cues that were informative with respect to target location. As in Experiment 1, a luminance control condition was included, and the participants performed a cue discrimination task with both luminance and isoluminance cues, in addition to the spatial cuing task.

\section{Method}

Participants. There were 18 adult volunteers in the luminance condition and 18 participants in the isoluminance condition.

Apparatus. The experiment was performed using an IBMcompatible 486 PC, together with a 15 -in. color monitor. The testing was performed in a sound-attenuated dimly lit room. Since the participants were tested under lower levels of ambient illumination, the brightness control of the monitor was reduced in comparison with Experiment 1 (see the luminance values below). A chinrest was used to control viewing distance at approximately $53 \mathrm{~cm}$.

Display and Stimuli (luminance condition). The display and stimuli were similar to those in Experiment 1. In Experiment 2, the gray outline boxes that were permanently present on the display were $1 \mathrm{~mm}$ ( 2 pixels) thick. In this experiment, the cue stimulus consisted of the inscription of an extra vertical gray line (1 pixel in thickness) on the inner side and immediately adjacent to the outer vertical of the left or the right square. This line was drawn in the same gray level (30 units) as that used for the fixation cross, outline squares, and target stimulus. The measured luminance of these stimuli was $4 \mathrm{~cd} \mathrm{~m}^{-2}$. As in Experiment 1, cue duration was $66 \mathrm{msec}$.

Display and Stimuli (isoluminance condition). The spatial dimensions of the stimuli in this condition were the same as those for the luminance condition. As in Experiment 1, the yellow display background was formed by combining 50 units of red with 50 units of green (luminance $=28 \mathrm{~cd} \mathrm{~m}^{-2}$ ). The brighter yellow used for the fixation cross, the target, and the 1-mm outline squares was formed by combining 56 units of red with 56 units of green (luminance = $\left.35 \mathrm{~cd} \mathrm{~m}^{-2}\right)$. The cue stimulus was formed by the addition of 35 units of blue (luminance $=36 \mathrm{~cd} \mathrm{~m}^{-2}$ ) along a vertical line ( 1 pixel in thickness) on the inner side and immediately adjacent to the outer vertical of the left or the right square.

Procedure (spatial cuing task). This procedure was similar to that in Experiment 1. The participants performed 12 practice trials followed by two blocks of 88 trials.

Procedure (cue discrimination task). This procedure was similar to that in Experiment 1. Although there was no manipulation of cue level in the spatial cuing task, in the cue discrimination task the participants were presented with three different levels of cues. In the luminance condition, this was achieved by drawing the cue stimulus in one of three different gray levels: 28 units (Cue Level One), 30 units (Cue Level Two), and 32 units (Cue Level Three). Hence, the cues employed in the spatial cuing task were identical to the Level Two cues in the cue discrimination task. In the isoluminance condition, cue level was manipulated by varying the number of blue units in the cue stimulus. The values used were 32 blue units (Cue Level One), 35 blue units (Cue Level Two), and 40 blue units (Cue Level Three). As in the luminance condition, the cues used in the spatial cuing task were identical to the Level Two cues in the cue discrimination task. In both the luminance and the isoluminance conditions, the participants performed 8 practice trials, followed by three blocks of 48 trials.

Design (spatial cuing task). The experiment comprised 160 target trials and 16 catch trials. There were equal numbers of trials with 


\section{Luminance Cues}

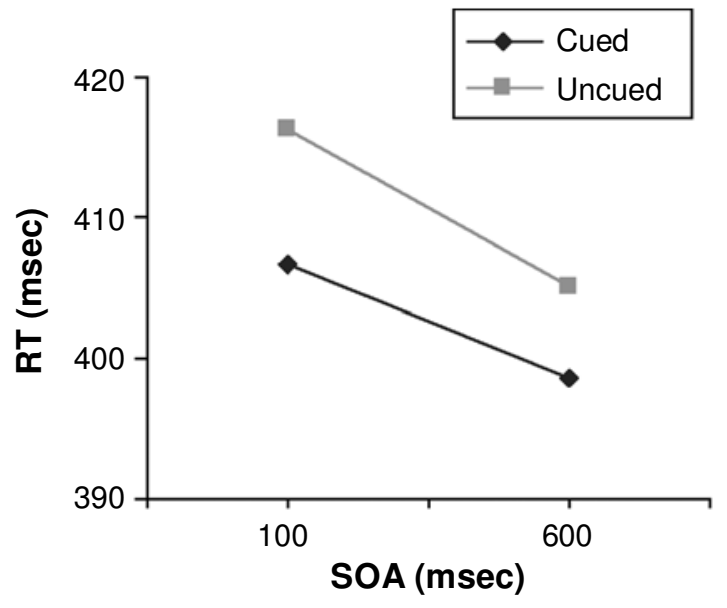

Figure 3. Response time (RT) results for the luminance condition in Experiment 2.

left and right cues ( 88 each), left and right targets (80 each), and 100and $600-$ msec SOAs ( 80 each). There were 128 trials on which the target was presented on the same side as the cue (cued trials) and 32 trials on which the cue and the target were presented on opposite sides (uncued trials). Trial type varied pseudorandomly with respect to all these attributes.

Design (spatial cuing task). This design was similar to that in Experiment 1.

\section{Results}

Spatial cuing task: Luminance condition. The mean rate of catch trial errors in this condition was $1.0 \%$. Response times less than $100 \mathrm{msec}$ in latency or greater than three standard deviations from the mean response time for that participant were excluded from further analysis. As a result of this procedure, $1.8 \%$ of the response times were discarded.

The response time results for the luminance condition of the spatial cuing task are shown in Figure 3. These data were entered into a two-factor analysis of variance for repeated measures. The factors were cuing (cued location vs. uncued location) and SOA (100 vs. $600 \mathrm{msec}$ ). Response latencies were shorter in the 600-msec SOA condition $(402 \mathrm{msec})$ than in the $100-\mathrm{msec}$ SOA condition [411 msec; $F(1,17)=10.67, p<.005]$. In addition, response latencies were shorter on cued trials $(403 \mathrm{msec})$ than on uncued trials [411 msec; $F(1,17)=5.00, p<.04]$. This cuing effect did not interact with SOA $(F<1)$.

Spatial cuing task: Isoluminance condition. The mean rate of catch trial errors in this condition was $0.35 \%$. Response times less than $100 \mathrm{msec}$ in latency or greater than three standard deviations from the mean response time for that participant were excluded from further analysis. As a result of this procedure, $1.8 \%$ of the response times were discarded.

The response time results for this condition are shown in Figure 4. These data were entered into an analysis of variance, with the same factors as those for the luminance condition. Response times were shorter in the 600-msec SOA condition $(339 \mathrm{msec})$ than in the $100-\mathrm{msec}$ SOA condition [350 msec; $F(1,17)=15.45, p<.005]$. Neither the main effect of cuing nor its interaction with SOA approached significance $(F<1)$.

Cue discrimination task: Luminance and isoluminance conditions. The participants' accuracy in the cue discrimination task is shown in Table 2. These data were entered into an analysis of variance with one repeated measures factor (cue level: One, Two, or Three) and one independent groups factor (cue condition: luminance cue vs. isoluminancecue). The main effect of cue level $[F(2,68)=$ $95.65, p<.001]$ was highly significant. There was no main effect of cue condition $(F<1)$, but this factor did interact with cue level $[F(2,68)=12.73, p<.001]$. As Table 2 shows, this interaction stems from the fact that there was a steeper increase of performance as a function of cue level in the luminance condition than in the isoluminance condition. However, it is also worth noting that at Cue Level Two, which was the level employed in the spatial cuing task, very similar levels of performance were obtained in the luminance $(82.3 \%$ correct $)$ and the isoluminance $(83.5 \%$ correct) conditions.

\section{Discussion}

The results from Experiment 2 show that even with cues that were informative regarding target location, isoluminant peripheral color changes had no effect on the orientation of attention. In contrast, a familiar pattern of results was obtained with peripheral cues involving a luminance change: Response times were quicker for targets appearing at the cued location, at both the short (100-msec) and the long $(600-\mathrm{msec})$ SOAs. This pattern is consistent with other reported results from investigations of the attentional effects of informative peripheral cues (Lambert et al., 1987).

The results from Experiments 1 and 2 show a fairly striking dissociation between the attentional effects of pe-

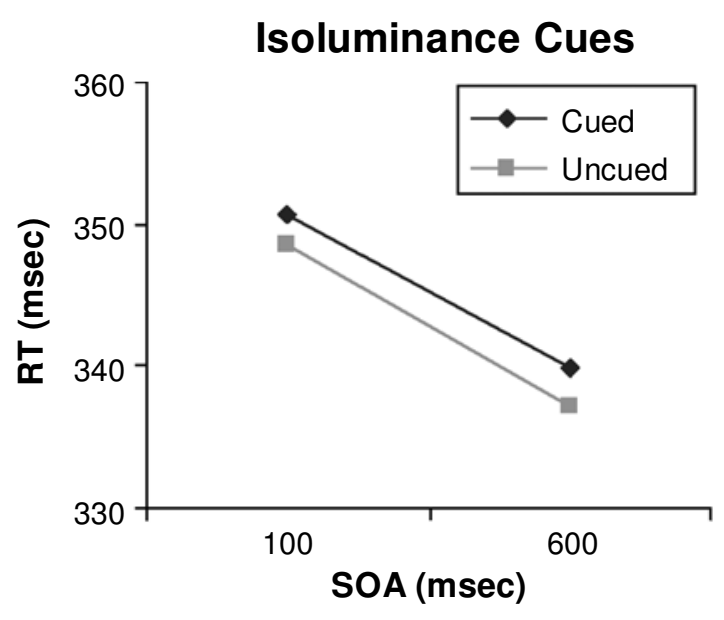

Figure 4. Response time (RT) results for the isoluminance condition in Experiment 2. 
Table 2

Cue Discrimination Accuracy (Percent Correct) in Experiment 2

\begin{tabular}{|c|c|c|c|c|}
\hline \multirow[b]{2}{*}{ Level } & \multicolumn{2}{|c|}{ Isoluminance Cues } & \multicolumn{2}{|c|}{ Luminance Cues } \\
\hline & $M$ & $S D$ & $M$ & $S D$ \\
\hline One & 74.2 & 15.1 & 61.9 & 13.0 \\
\hline Two & 83.4 & 17.0 & 82.3 & 14.3 \\
\hline Three & 88.3 & 16.3 & 92.1 & 7.6 \\
\hline
\end{tabular}

ripheral luminance cues and of isoluminant peripheral color changes. Theoretically, one possible interpretation of this pattern is to propose that the rapid shifts of attention, indexed by the cuing effects observed in these experiments, are controlled, or at least initiated, exclusively within the luminance sensitive dorsal pathway. This will be referred to below as the dorsal pathway hypothesis. According to this interpretation, isoluminant cue stimuli, processed entirely within the ventral system, do not trigger attention shifts, because they do not gain access to the dorsal pathway.

However, research carried out by Charles Folk and associates (Folk, Remington, \& Johnston, 1992) suggested an alternative interpretation. Folk et al. (1992) reported a series of experiments showing that the attentional effects of peripheral cue stimuli varied according to the attentional control settings adopted by the participants in response to specific task demands. When the participants searched for a target defined by an abrupt onset (luminance change), a peripheral cue that also had this property captured attention, but a peripheral color cue did not. Conversely, when the participants searched for a target defined by a color change, a peripheral color cue captured attention, but a peripheral abrupt onset did not. Unfortunately, since the color cues and targets used by Folk et al. (1992) were not isoluminant with the background, their data did not address directly the issue of whether isoluminant stimuli can capture attention. Nevertheless, their experiments provided elegant evidence that the propensity of peripheral stimuli to capture attention can be modulated by the task set adopted by participants. This point is strongly relevant here, since the target stimulus in both Experiment 1 and Experiment 2 involved a change in display luminance. In the luminance conditions, the target appeared as a gray square against a black background, whereas in the isoluminance conditions, the target was a bright yellow square against a yellow background. Hence, according to the contingent involuntary orienting hypothesis (CIOH) of Folk et al. (1992) the absence of attentional capture in our isoluminance conditions can be explained by the argument that, in these conditions, target and cue stimuli did not share a defining property and that the attentional set adopted by the participants precluded attentional capture by isoluminant color changes. Experiments 3 and 4 were designed to discriminate between the $\mathrm{CIOH}$ and the dorsal pathway hypothesis.

However, before describing these experiments, two further aspects of the results from Experiments 1 and 2 merit discussion. It is interesting and surprising that in the isoluminance condition of Experiment 2, there was no advantage for cued over uncued trials even at the long SOA. As was mentioned earlier, in previous studies, informative peripheral cues have produced cued side advantages at both short and long SOAs (Lambert et al., 1987), and this has been interpreted in terms of the combined effects of exogenous orienting and endogenous orienting, influencing performance at short and long SOAs, respectively. Given this interpretation, the absence of an advantage in the isoluminance condition for cued trials at the long SOA is surprising. Data from the cue discrimination task show that left-sided cues can easily be discriminated from rightso why do participants fail to orient under endogenous/ voluntary control to the cued side? This is indeed puzzling. One possible solution involves appealing to the spatial correspondence hypothesis of Lambert and Duddy (2002). These authors used bilateral letter pairs as spatial cues and found that spatially asymmetric cue displays (e.g., $\mathrm{X}+\mathrm{T}$ ) produced faster and stronger orienting than did spatially symmetric (e.g., $\mathrm{X}+\mathrm{X}$ ) cues. On the basis of these results, we derived the spatial correspondence hypothesis, which proposes that the speed and strength of orienting in response to both central and peripheral cues are critically related to the degree of spatial correspondence between features of the cue display and features of the target display. This is high in peripheral cuing experiments in which there is a clear correspondence between the location of the cue stimulus and the location of the target. Spatial correspondence is lower but still present in experiments that employ central cues, such as arrowheads. This idea has been developed further by Wells (2000), who described an experiment in which single centrally presented letters were used as spatial cues. In this experiment, significant orienting effects were observed in a condition in which the letter cues were spatially asymmetric ( $d$ as a left cue, $b$ as a right cue); but in a condition in which the letter cues were perfectly symmetric ( $x$ as a right cue, $o$ as a left cue), orienting effects were absent. This was interpreted in terms of the spatial correspondencehypothesis, since in the former condition the participants were able to form associative links between target location and spatial features of the cue letters $d$ and $b$, whereas with symmetric letters, such as $x$ and $o$, this was impossible. Hence, in both this study and in the isoluminance condition of the present experiment, participants failed to orient despite being provided with clear cue information indicating a left or a right target. In both cases, this failure may have been due to an inability to form associative links between spatial features of the cue and target displays. However, in the isoluminance condition of the present experiment, this failure may have been due not to an absence of asymmetry per se in the target display, but to an absence of asymmetry encoded within the dorsal stream (if the dorsal stream hypothesis is assumed) or to an absence of asymmetry in the perceptual domain(s) appropriate to the current attentional control setting (if the contingent orienting hypothesis is assumed). 
A final issue for discussion is methodological and bears on interpretation of the results from Experiment 1, as well as those from Experiment 2. In both experiments, the cue discrimination task allowed us to compare the relative salience of the cue stimuli employed in the luminance and the isoluminance conditions. This allowed us to justify the claim that the isoluminance cues were at least as salient as the luminance cues. However, the target stimuli were somewhat different in the two conditions, since these consisted of a gray square presented against a black background and a bright yellow square against a yellow background in the luminance and the isoluminance conditions, respectively. This raises the possibility that the differences we observed in the propensity of luminance and isoluminance cues to capture attention might have been driven by differences in the perceptual salience of the targets, rather than by properties of the cue stimuli. That is, there is a potential confound between target salience and the cue property of luminance or isoluminance. This issue is easily disposed of. Inspection of the overall mean response times in the luminance and isoluminance conditions of Experiments 1 and 2 (see Figures 1-4) shows the following. In Experiment 1 , mean response latency to targets in the luminance condition was $314 \mathrm{msec}$, whereas mean latency in the isoluminance condition was $347 \mathrm{msec}$. The contrast between these two conditions was not significant $[t(14)=1.47$, n.s.]. Hence, in Experiment 1, which was performed with normal levels of room illumination, target salience, as indexed by overall response time, was similar in the two conditions. In contrast, in Experiment 2, which was performed with a lower level of ambient illumination (see the Method section), response latencies were significantly longer in the luminance condition $(407 \mathrm{msec})$ than in the isoluminance condition [345 msec; $t(34)=3.88, p<$ .001]. The most likely source of this difference is the lower level of target luminance employed in the luminance condition of Experiment 2 (see the Method section). Since the relative salience of targets in the luminance and the isoluminance conditions was quite different in Experiments 1 and 2, we can discount the possibility that the common pattern observed in these experiments (i.e., attentional capture by luminance cues, no attentional capture by isoluminance cues) was driven by differences in target salience, rather than by cue properties.

\section{EXPERIMENT 3}

In Experiment 3, the participants were presented with peripheral luminance cues and they responded to the onset of a target that was defined by an isoluminant color change. In a sense, this represents the converse of the situation in Experiments 1 and 2, in which the participants were presented with isoluminance cues and responded to targets that were accompanied by a luminance change. In both situations, the defining properties of the cue and the target were discordant. According to the $\mathrm{CIOH}$, similar results should be obtained in both situations. That is, peripheral luminance changes should fail to capture atten- tion when participants are searching for a target defined by an isoluminant color change.

\section{Method}

Participants. Twelve adult volunteers took part.

Apparatus. The apparatus was the same as that for Experiment 2.

Display and Stimuli. The display and stimuli were the same as those in Experiment 1 (isoluminance condition), with the following exceptions. The two outline squares that were permanently present on the screen were presented in gray (gray level $=30$ ) against a yellow background (which was formed by combining 50 units of red with 50 units of green). The cue stimuli were formed by presenting a thin outline square (1-mm thickness, 2 pixels) in a slightly lighter gray superimposed over and against the background of one of the permanently present gray outline squares. As in Experiment 1, cues with three different levels of intensity were presented. These were formed by presenting the thin light gray square with a gray level of 32 (Cue Level One), 35 (Cue Level Two), or 40 (Cue Level Three). The target stimulus was of the same dimensions as in Experiments 1 and 2 but was formed by adding 35 units of blue to the yellow background.

Procedure (spatial cuing task). This procedure was the same as that in Experiment 1.

Procedure (cue discrimination task). This procedure was the same as that in Experiment 1.

Design (spatial cuing task and cue discrimination task). The design was the same as that in Experiment 1.

\section{Results}

Spatial cuing task. The mean rate of catch trial errors in this condition was $0.9 \%$. Response times less than $100 \mathrm{msec}$ in latency or greater than three standard deviations from the mean response time for that participant were excluded from further analysis. As a result of this procedure, $2.0 \%$ of response times were discarded.

The response time results for the spatial cuing task are shown in Figure 5. These data were entered into a threefactor analysis of variance for repeated measures. The factors were the same as those in the analyses of the data from Experiment 1. As in the earlier experiments, response latencies were shorter in the 600-msec SOA condition (429 msec) than in the 100-msec SOA condition [451 msec;

\section{Luminance Cues}

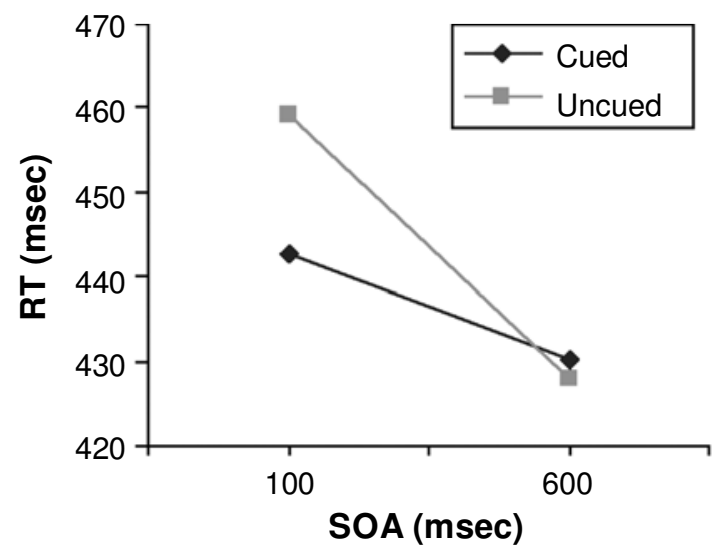

Figure 5. Response time (RT) results for Experiment 3. 
Table 3

Mean Response Latency (in Milliseconds) in Experiment 3

\begin{tabular}{lccccc}
\hline & \multicolumn{2}{c}{ SOA $100 \mathrm{msec}$} & & \multicolumn{2}{c}{ SOA 600 msec } \\
\cline { 2 - 3 } \cline { 5 - 6 } Level & Cued & Uncued & & Cued & Uncued \\
\hline One & 446 & 458 & & 438 & 430 \\
Two & 447 & 452 & & 423 & 433 \\
Three & 435 & 468 & & 431 & 421 \\
\hline
\end{tabular}

$F(1,11)=9.79, p<.02]$. The main effect of cuing was significant $[F(1,11)=5.18, p<.05]$. However, this effect also entered into two interactions. There was an interaction between cuing and SOA $[F(1,11)=12.67, p<.005]$. At the short SOA, response latencies were shorter on cued trials $(443 \mathrm{msec})$ than on uncued trials $(459 \mathrm{msec})$, whereas at the long SOA, cued $(430 \mathrm{msec})$ and uncued $(428 \mathrm{msec})$ trials did not differ. In addition, there was a three-way interaction between cuing, SOA, and level $[F(2,22)=4.68, p<$ .025]. A full breakdown of this interaction is shown in Table 3. Simple effects analyses showed that the 33-msec advantage for cued over uncued trials at the short SOA with Level 3 cues was significant $[F(1,11)=17.09, p<$ .005]. However, the differences between cued and uncued trials at the short SOA with Level 2 cues $(5 \mathrm{msec})$ and with Level 1 cues $(12 \mathrm{msec})$ did not attain significance.

Cue discrimination task. The participants' accuracy in the cue discrimination task is shown in Table 4. These data were entered into a one-way analysis of variance. The main effect of cue level was significant $[F(2,22)=4.04$, $p<.05]$. Discrimination performance with Cue Level One $(92.4 \%)$ was somewhat inferior to performance with Cue Levels Two (97.1\%) and Three (97.6\%).

\section{Discussion}

The results from this experiment appear to be inconsistent with the contingent attentional capture hypothesis, since peripheral luminance changes captured attention even when the participants responded to a target defined by an equiluminant color change. Conversely, the results are consistent with the view that processing of abrupt luminance changes within the dorsal pathway plays an important role in the control of visual orienting. However, the conditions making up this experiment (luminance cues paired with isoluminant targets) do not provide an exhaustive test of predictions that can be derived from the $\mathrm{CIOH}$. The latter also predicts that when participants are searching for an isoluminant target, isoluminant visual changes should capture attention. Accordingly, further discussion of the present experiment will be deferred until the results from a final experiment have been described.

\section{EXPERIMENT 4}

In Experiment 4, we tested a further prediction derived from the $\mathrm{CIOH}$ regarding orienting in response to peripheral cues. This hypothesis predicts that, in contrast to the results obtained from Experiments 1 and 2, peripheral cues consisting of an isoluminant color change will cap- ture attention when the target stimulus is also defined by this property.

\section{Method}

Participants. Eight adult volunteers took part.

Apparatus. The apparatus was the same as that for Experiments 2 and 3 .

Display and Stimuli. The display and stimuli were the same as those for Experiment 3, with the following exception. The cue stimuli were identical to those employed in the isoluminance condition of Experiment 1.

Procedure (spatial cuing and cue discrimination tasks). The procedure was the same as that for Experiment 3.

Design (spatial cuing and cue discrimination tasks). The design was the same as that for Experiment 3.

\section{Results}

Spatial cuing task. The mean rate of catch trial errors in this condition was $1.4 \%$. Response times less than $100 \mathrm{msec}$ in latency or greater than three standard deviations from the mean response time for that participant were excluded from further analysis. As a result of this procedure, $3.0 \%$ of the response times were discarded.

The response time results for the spatial cuing task are shown in Figure 6. These data were entered into a threefactor analy sis of variance for repeated measures. The factors were the same as those in the analyses of the data from Experiment 1 . As in the earlier experiments, response latencies were shorter in the $600-\mathrm{msec}$ SOA condition $(424 \mathrm{msec})$ than in the 100-msec SOA condition [457 msec; $F(1,7)=$ $13.75, p<.01]$. The main effect of cue level was significant $[F(1,7)=5.43, p<.05]$. This showed that response latencies were somewhat longer following Level 1 cues (447 msec) than following Level 2 (438 msec) and Level 3 $(437 \mathrm{msec})$ cues. The main effect of cuing was significant $[F(1,7)=16.22, p<.01]$, as was the interaction between cuing and SOA $[F(1,7)=16.46, p<.01]$. As Figure 6 shows, this interaction arose from the fact that at the short SOA, response latencies were shorter on cued trials (448 msec) than on uncued trials (466 msec), whereas at the long SOA, cued $(423 \mathrm{msec})$ and uncued $(425 \mathrm{msec})$ trials did not differ. No further effects were significant in this analysis.

Cue discrimination task. The participants' accuracy in the cue discrimination task is shown in Table 4. These data were entered into a one-way analysis of variance. The main effect of cue level was significant $[F(2,14)=3.97$, $p<.05]$. Discrimination performance with Cue Level

Table 4

Cue Discrimination Accuracy (Percent Correct) in Experiments 3 and 4

\begin{tabular}{|c|c|c|c|c|}
\hline \multirow[b]{2}{*}{ Level } & \multicolumn{2}{|c|}{$\begin{array}{c}\text { Experiment } 3 \\
\text { (Luminance Cues) }\end{array}$} & \multicolumn{2}{|c|}{$\begin{array}{c}\text { Experiment } 4 \\
\text { (Isoluminance Cues) }\end{array}$} \\
\hline & $M$ & $S D$ & $M$ & $S D$ \\
\hline One & 92.4 & 11.0 & 92.4 & 9.5 \\
\hline Two & 97.0 & 6.4 & 96.6 & 5.0 \\
\hline Three & 97.6 & 4.9 & 98.2 & 3.0 \\
\hline
\end{tabular}


Isoluminance Cues

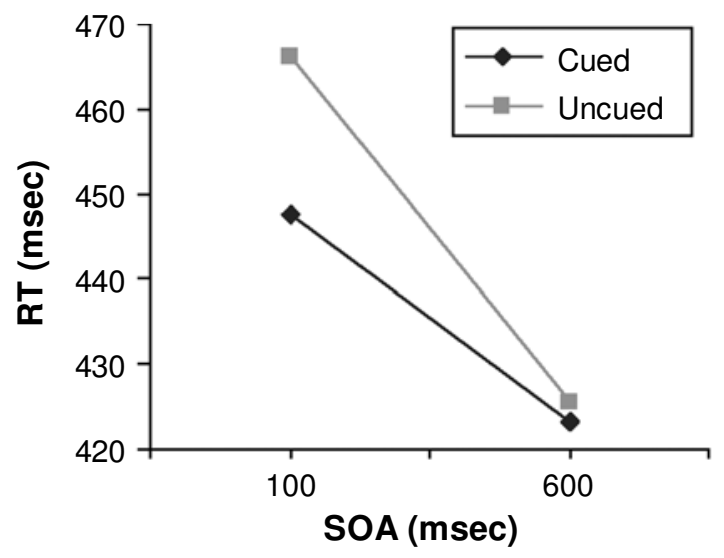

Figure 6. Response time (RT) results for Experiment 4.

One $(92.5 \%)$ was somewhat inferior to performance with Cue Levels Two (96.6\%) and Three (98.2\%).

\section{Discussion}

Unlike Experiment 3, this experiment produced results consistent with the $\mathrm{CIOH}$. As predicted by this hypothesis, isoluminant peripheral color changes captured attention when the participants searched for a peripheral target that was also signaled by an isoluminant color change. This is an important result, since it shows that the mechanisms that subserve the attentional capture effect are not the sole province of neural structures and processes within the luminance-sensitive dorsal pathway. This may be taken to imply that the mechanisms responsible for attentional capture are represented at multiple brain locations, which include both the luminance-sensitive dorsal pathway and the color-sensitive ventral pathway. Furthermore, as was hypothesized by Folk et al. (1992), it appears that the propensity of visual stimuli to capture attention is modulated by the task set adopted by participants.

\section{GENERAL DISCUSSION}

The results from the experiments reported above show that under appropriate task conditions, isoluminant color changes can indeed capture attention. In addition, Experiments 3 and 4 shed further light on the CIOH of Folk et al. (1992). These findings provided mixed support for $\mathrm{CIOH}$, and in interpreting them, it will be useful to distinguish between strong and weak versions of the hypothesis. According to the strong version of $\mathrm{CIOH}$, the propensity of any stimulus to capture attention is directly related to the specific attentional set adopted by participants, which is, in turn, determined by the particular demands of the task at hand. Hence, a peripheral cue defined by luminance change will capture attention only when participants adopt a task set that renders the attentional system sensitive to luminance change. Similarly, a peripheral cue defined by color change will capture attention only in the context of an appropriate color-sensitive attentional set.
Clearly, the argument also applies to other dimensions, such as stimulus motion or binocular disparity. However, a weaker version of $\mathrm{CIOH}$ can also be advanced. This version of the hypothesis proposes that the tendency of peripheral visual stimuli to capture attention is modulated by task demands but also acknowledges that certain dimensions of stimulus change-particularly, luminance change-may play an especially important role in mediating attentional capture across a variety of task settings.

Taken together, the findings from the present study are consistent with this weak version of $\mathrm{CIOH}$. The results from Experiments 1, 2, and 4 certainly support the view that task set is a crucial factor modulating attentional capture by peripheral stimuli. In Experiments 1 and 2, isoluminant peripheral cues failed to capture attention when the participants were set to detect a target stimulus that included a luminance change. In Experiment 4, isoluminant color change cues did capture attention when both the cues and the targets were defined in terms of this stimulus dimension. These findings are consistent with a variety of other studies that have reported effects of task set on attentional capture by peripheral cue stimuli. In addition to the findings of Folk et al. (1992) described previously, Yantis and Jonides (1990) found that when attention was strongly focused on a single location, an abrupt onset (luminance) cue presented elsewhere failed to capture attention. In addition, Lambert et al. (1987) found that a peripheral luminance cue failed to capture attention when participants were explicitly informed that targets usually appeared at a display location on the side opposite to the cue stimulus.

Although the results from Experiments 1, 2, and 4 are generally consistent with $\mathrm{CIOH}$, the results from Experiment 3 appear to be difficult to reconcile with the strong version of this proposal. In Experiment 3, since targets were defined solely in terms of an isoluminant color change, there was no incentive for the participants to adopt an attentional control setting in which rapid attention shifts are triggered via luminance change. Nevertheless, there was clear evidence of attentional capture by luminance cues in this experiment. Hence, it appears that the relation between attentional control settings and dimensions of cue stimulus variation (i.e., color change or luminance change) is somewhat asymmetric. Isoluminant color changes captured attention when the participants detected a target that was also defined by this property, but they failed to capture attention when the participants detected a luminance change target. However, luminance-based cues captured attention regardless of whether the participants' task was to detect a luminance change target or an isoluminant color change target. This pattern of results appears to be inconsistent with the strong version of $\mathrm{CIOH}$ but is consistent with a weaker version of this hypothesis, which acknowledges that luminance changes may play an especially important role in triggering rapid shifts of attention. This would certainly make sense from an evolutionary point of view, since in most natural environments, the appearance of important survival-related objects (e.g., potential prey or predators) in peripheral vision will be accom- 
panied by variations in luminance. Conversely, situations in which the appearance of important peripheral objects is signaled by color change with little or no change in luminance are probably quite rare. In discussing their own results, Folk et al. (1992) appear to have acknowledged this point and suggested that "when there is little incentive to configure the system for any other property, abrupt luminance change ... may be instantiated as the "default' setting" (see also Folk, Remington, \& Johnston, 1993; Lambert et al., 1987; Yantis, 1993). The point made here is perhaps an important one: It appears that the attentional system will respond to luminance change unless there is a substantial disincentive to do so. In the appropriate conditions of Experiments 1 and 3 of Folk et al. (1992) and in the informed uncued probable condition of Lambert et al. (1987), targets either never appeared at the cued location or usually $(p=.75$ or $p=.80)$ appeared at an uncued location (see also Theeuwes, 1991; Yantis \& Jonides, 1990). Under all these task conditions, it was distinctly advantageous to either suppress orienting toward the cue or use the cue as a signal to orient elsewhere. In Experiment 3 of the present study, (luminance) cue location and target location were uncorrelated; therefore, there was no strong incentive to suppress the "default" setting at which luminance change triggers a shift of attention. In light of these points, it could be argued that the conditions embodied in Experiment 3 provided a relatively weak test of $\mathrm{CIOH}$, since there was little incentive for the participants to suppress the "default setting" at which luminance changes capture attention. Therefore, when considered together, the results from Experiments 3 and 4 may be taken as consistent with a version of $\mathrm{CIOH}$ in which luminance changes play an especially important role in triggering attention movements.

The asymmetry that we observed with respect to the attention-capturing properties of luminance cues and isoluminant color cues can be viewed from two perspectives. In terms of the neurological substrates of visual orienting, the fact that luminance cues captured attention under both task sets (i.e., for luminance targets [Experiments 1 and 2] and for isoluminant targets [Experiment 3]), whereas isoluminance cues captured attention only with a task set for isoluminant targets (Experiment 4), can be seen as corresponding to broader sensitivity to luminance signals in the visual pathways - that is, the dorsal and ventral pathways are both sensitive to luminance change, whereas isoluminant color signals are processed solely within the ventral pathway. A second perspective, which is complementary with rather than exclusive of the first, is in terms of the time-course of processing luminance signals and isoluminant color signals. In Experiments 1 and 2, the participants made a simple detection response to targets that included a luminance signal. The mean latency for detecting these targets was $359 \mathrm{msec}$. In Experiments 3 and 4, the participants made a simple detection response to a target defined by an isoluminant color change. Mean detection latency in these experiments was $440 \mathrm{msec}$, which was clearly slower than those in Experiments 1 and $2[t(70)=$ $5.63, p<.001]$. This finding is, of course, consistent with known characteristics of luminance and color processing in the dorsal and the ventral pathways, respectively (see, e.g., Livingstone \& Hubel, 1988). Fast processing of luminance signals, together with slow processing of isoluminant color signals, is also consistent with our findings concerning attentional capture. Since isoluminant color signals are processed relatively slowly, there may have been little or no opportunity for cue processing to influence target processing in the short-SOA isoluminance conditions of Experiments 1 and 2. Processing of the luminance-based targets in these experiments may have been effectively complete prior to encoding of the isoluminance cues. This would not have been the case in the short-SOA condition of Experiment 4, in which processing of both the cue and the target involved relatively slow encoding of an isoluminant color signal. Conversely, in the short-SOA condition of Experiments 1-3, there may have been plenty of opportunity for rapidly encoded luminance cue information to interact with either a rapidly encoded luminance target signal (Experiments 1 and 2) or a more slowly encoded isoluminant target signal (Experiment 4).

As was mentioned earlier, our observation of attentional capture in Experiment 4 can be taken as evidence that the processes responsible for this effect can be mediated within the ventral, as well as the dorsal, pathway. If this is accepted, two alternative interpretations for the results of Experiment 3 become plausible. In this experiment, luminance cues captured attention in a task in which participants detected an isoluminant target. Following our earlier interpretation, it is assumed that detection of the isoluminant target is achieved within the ventral pathway. If the ventral pathway can mediate attentional capture, and since the ventral pathway is sensitive to both luminance and color, attentional capture in Experiment 3 could have been mediated in either of two ways: by interaction between ventral processing of the target and ventral processing of the luminance signal provided by the cue or by interaction between ventral processing of the target and dorsal processing of the cue luminance signal.

Astute readers may also notice that, in principle, a third possibility could also be entertained: that attentional capture in Experiment 3 was mediated by interaction between ventral processing of the target and ventral processing of a color signal generated by the cue. The cues employed in this experiment consisted of a thin light gray line presented against a dark gray frame. The subjective appearance of this was that the gray frame appeared to flash briefly (i.e., change in luminance), without any apparent change in color. Nevertheless, it is conceivable that a change of luminance within the gray part of the screen might have generated a color signal within the ventral pathway, because of a transient change in the simultaneous color contrast between this gray region and the expanse of yellow that occupied most of the screen. Although this cannot be ruled out, such a color signal would have been slight indeed, in comparison with the clear luminance signal provided by cue onset and offset.

In conclusion, the results of this study show that under appropriate conditions, isoluminant color changes can 
capture attention. This finding suggests that the neural processes underlying attentional capture by peripheral visual changes are not mediated solely within the dorsal pathway. Rather, it appears that attentional capture may be mediated at multiple brain sites, involving both the colorsensitive ventral pathway and the luminance-sensitive dorsal pathway. The results also show that the attentional control setting adopted by participants is an important factor that determines whether isoluminant stimuli will or will not capture attention. Our findings were in broad agreement with the CIOH of Folk et al. (1992), since it was found that isoluminant color changes captured attention only when the participants adopted an appropriate color-sensitive task set, in which both the cue and the target were defined by isoluminant color change. However, the observation that luminance cues captured attention regardless of whether the participants were set to detect a luminance target or a color change target suggests that luminance change signals play an especially important role in triggering rapid shifts of attention.

\section{REFERENCES}

Cavanagh, P., Adelson, E., \& Heard, P. (1992). Vision with equiluminant contrast: 2. A large scale technique and observations. Perception, 21, 219-226.

Cheal, M., \& Lyon, D. (1991). Central and peripheral precuing of forced choice discrimination. Quarterly Journal of Experimental Psychology, 43A, 859-880.

Folk, C. L., Remington, R. W., \& Johnston, J. C. (1992). Involuntary covert orienting is contingent on attentional control settings. Journal of Experimental Psychology: Human Perception \& Performance, 18, 1030-1044.

Folk, C. L., Remington, R. W., \& Johnston, J. C. (1993). Contingent attentional capture: A reply to Yantis (1993). Journal of Experimental Psychology: Human Perception \& Performance, 19, 682-685.

Gellatly, A., Cole, G., \& Blurton, A. (1999). Do equiluminant object onsets capture visual attention? Journal of Experimental Psychology: Human Perception \& Performance, 25, 1609-1624.

JonIDES, J. (1981). Voluntary versus automatic control over the mind's eye's movement. In J. [B.] Long \& A. [D.] Baddeley (Eds.), Attention and performance IX (pp. 187-203). Hillsdale, NJ: Erlbaum.

LAmbert, A. [J.], \& DUDDY, M. (2002). Visual orienting with central and peripheral precues: Deconfounding the contributions of cue eccentricity, cue discrimination and spatial correspondence. Visual Cognition, 9, 303-336.

Lambert, A. [J.], Naikar, N., Mclachlan, K., \& Aitken, V. (1999). A new component of visual orienting: Implicit effects of peripheral information and subthreshold cues on covert attention. Journal of Experimental Psychology: Human Perception \& Performance, 25, 321-340.

Lambert, A. [J.], Spencer,E., \& Mohindra, N. (1987). Automaticity and the capture of attention by a peripheral display change. Current Psychological Research \& Reviews, 6, 136-147.

Lambert, A. J., Spencer, M. B. H., \& Hockey, G. R. J. (1991). Peripheral visual changes and spatial attention. Acta Psychologica, 76, 149-163.

Livingstone, M., \& Hubel, D. (1988). Segregation of form, color, movement, and depth: Anatomy, physiology, and perception. Science, 240, 740-749.

Luck, S. J., \& Thомas, S. J. (1999). What variety of attention is automatically captured by peripheral cues? Perception \& Psychophysics, 61, 1424-1435.

MaYlor, E. A. (1985). Facilitatory and inhibitory components of orienting in visual space. In M. I. Posner \& O. S. M. Marin (Eds.), Attention and performance XI (pp. 189-204). Hillsdale, NJ: Erlbaum.

Merigan, W. H., \& Maunsell, J. H. R. (1993). How parallel are the primate visual pathways? Annual Review of Neuroscience, 16, 369-402.
Muller, H., \& Rabbitt, P. M. A. (1989). Reflexive and voluntary orienting of visual attention: Time course of activation and resistance to interruption. Journal of Experimental Psychology: Human Perception \& Performance, 15, 315-330.

Posner, M. I., \& Cohen, Y. (1984). Components of visual orienting. In H. Bouma \& D. G. Bouwhuis (Eds.), Attention and performance $X$ : Control of language processes (pp. 531-556). Hillsdale, NJ: Erlbaum.

Pratt, J., Hillis, J., \& Gold, J. M. (2001). The effect of the physical characteristics of cues and target on facilitation and inhibition. Psychonomic Bulletin \& Review, 8, 489-495.

Robertson, L., \& RAFAL, R. (2000). Disorders of visual attention. In M. S. Gazzaniga (Ed.), The new cognitive neurosciences (2nd ed., pp. 633-649). Cambridge, MA: MIT Press, Bradford Books.

TheEuwES, J. (1991). Exogenous and endogenous control of attention: The effect of visual onsets and offsets. Perception \& Psychophysics, 49, 83-90.

TheEUWES, J. (1995). Abrupt luminance change pops out; abrupt color change does not. Perception \& Psychophysics, 57, 637-644.

Ungerleider, L. G., \& MishKin, M. (1982). Two cortical visual systems. In D. J. Ingle, M. A. Goodale, \& R. J. W. Mansfield (Eds.), Analysis of visual behavior (pp. 549-586). Cambridge, MA: MIT Press.

WELLS, I. (2000). The role of isoluminance and symmetry in visual orienting of attention. Unpublished master's thesis, University of Auckland.

YANTIS, S. (1993). Stimulus-driven attentional capture and attentional control settings. Journal of Experimental Psychology: Human Perception \& Performance, 19, 676-681.

Yantis, S., \& Hillstrom, A. P. (1994). Stimulus-driven attentional capture: Evidence from equiluminant visual objects. Journal of Experimental Psychology: Human Perception \& Performance, 20, 95-107.

YANTIS, S., \& JonIDES, J. (1990). Abrupt visual onsets and selective attention: Voluntary versus automatic allocation. Journal of Experimental Psychology: Human Perception \& Performance, 20, 95-107.

Zihl, J., von CrAmon, D., \& MAI, N. (1983). Selective disturbance of movement vision following bilateral brain damage. Brain, 106, 251-265.

\section{NOTES}

1. Readers will have noted that the values given here for the measured luminances of isoluminance cues are slightly less than the value given for the bright yellow frames, which formed the background to the cue. This might seem paradoxical, since the cues were formed by the addition of blue light to a yellow background. The reason for this paradox is that measured values for luminance attempt to take into account the spectral sensitivity of a human observer-luminance depends not only on physical energy, but also on the spectral composition of the light source. For example, a blue light source with the same physical energy as a yellow light source will have considerably lower luminance. In addition, the spectral sensitivity function itself varies according to overall levels of illumination. In the present case, it appears that although the physical energy of the cues was greater than that of the background, our photometer (Minolta LS11) registered an anomalous reduction in luminance, due to the increasing proportion of blue light in the cue stimuli. However, in perceptual terms, presentation of the isoluminance cues was not accompanied by any noticeable flicker.

2. Since it clear that there was a substantial effect of cue level on discrimination performance in the luminance condition, curiosity is prompted concerning the relation between luminance cue level and cuing effects in the spatial attention task. As was noted earlier, neither the interaction between cue level and cuing $(F<1)$ nor the three-way interaction between cue level, cuing, and SOA $[F(1,7)=1.09$, n.s. $]$ approached significance. However, detailed examination of the data suggests that the strongest contribution to the cuing $\times$ SOA interaction observed in the luminance condition came from performance with Level Three cues. When the data from this condition were examined separately, both the interaction between cuing and SOA $[F(1,7)=6.48, p<.05]$ and the advantage for cued $(305 \mathrm{msec})$ over uncued $(330 \mathrm{msec})$ trials at the short SOA $[F(1,7)=$ $9.38, p<.025]$ remained significant.

(Manuscript received March 16, 2000; revision accepted for publication August 8, 2002.) 